\title{
A Study of Economic Value Estimation on Cryptocurrency Value back by Gold, Methods, Techniques, and Tools
}

\author{
Ferdiansyah $^{1}$, Siti Hajar Othman², Raja Zahilah Md Radzi ${ }^{3}$, \\ Deris Stiawan 4
}

\author{
${ }^{1}$ Informatics Department, Universitas Bina Darma, Palembang, Indonesia \\ 2,3School of Computing, Faculty of Engineering, Universiti Teknologi Malaysia, Johor Bahru, \\ Johor, Malaysia \\ ${ }^{4}$ Faculty of Knowledge, Universitas Sriwijaya, Bukit Besar, Palembang, Indonesia

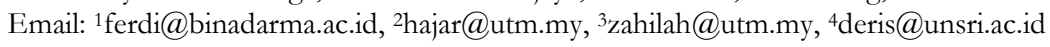

\begin{abstract}
After Bitcoin Introduced around the world, many Cryptocurrencies was created that followed the standard of bitcoin. The use of Bitcoin or other Cryptocurrency as a currency is also an interesting study from an Islamic economic perspective. They tried to use gold with value back by gold, which gold itself is famous for its exchange rate stability. From abu bakar There is a need for monitoring organization of the cryptocurrency, to controlling from Riba (Interest), Maysir (gambling) and ghahar (Uncertainty). To solve this problem there is a need a tool that can predict with certainty based on valid historical data, to produce accurate prediction results and produce Economic value estimations that are close to Gold real value. With the results we can monitoring day by day, see next day value and continuously based on Cryptocurrency with value back by gold, and see what other impact influences the value by looking the factor negative or positive with sentiment analysis. In the last section we discuss and provide method that we analyse from previous work to produce method to estimate value cryptocurrency value back by gold.
\end{abstract}

Keywords: Bitcoin, Cryptocurrency, Economic Value Estimation, Prediction, Shariah Compliance.

\section{INTRODUCTION}

The cryptocurrency is a new emerging popular topic to be discussed from all around the world, both in its adoption, its application in life, technology and, safety which is still being studied and studied thoroughly and research on cryptocurrency is also growing along with its development. Recently, cryptocurrency has attracted extensive attentions from both industry and academia. Bitcoin that is often called 


p-ISSN: 2656-5935 http://journal-isi.org/index.php/isi e-ISSN: 2656-4882

the first cryptocurrency has enjoyed huge success with the capital market reaching 10 billion dollars in 2016[1].

Nowadays after Bitcoin Introduced around the world, many Cryptocurrencies was created that followed the standard of bitcoin. The use of Bitcoin or other Cryptocurrency as a currency is also an interesting study from an Islamic economic perspective. There is another problem as we mention above from abu bakar There is a need for monitoring organization of the cryptocurrency [2], to controlling from Riba (Interest), Maysir (gambling) and ghahar (Uncertainty)[3]. To solve this problem there is a need a tool that can predict with certainty based on valid historical data, to produce accurate prediction results and produce Economic value estimations that are close to Gold real value. With the results we can monitoring day by day, see next day value and continuously based on Cryptocurrency with value back by gold, and see what other impact influences the value by looking the factor negative or positive with sentiment analysis.

\section{LITERATURE REVIEW}

\subsection{History of Cryptocurrency}

The history of cryptocurrency (Cryptographic currency) begins in the 1980s started with David chaum, In his paper, he proposed a novel of a cryptographic scheme to blind the content of the message before it is signed so that the signer cannot determine the content. These blind signatures can be publicly verified just like a regular digital signature. Chaum proposed digital cash approach in such a way that is untraceable by another party.[4]

The rise of cryptocurrency started on B-money In 1998, Wei Dai proposed bmoney[5], an anonymous and distributed electronic cash system, In that method, describes two protocols based on network that cannot be traced, where senders and receivers are identified only by digital such as their public keys, and each message will be signed by its sender to receiver.

Bit Gold In 1998, Nick Szabo[6] propose models a new digital currency, the models based on cryptographic system puzzles, which after being solved, were sent to the Byzantine-fault-tolerant public registry and assigned to the public key of the solver.

Hashcash proposed by Adam Back, Haschash, a system relied on a cryptographic hash function to derive a probabilistic proof of computational work as authentification system Pow (Proof of work)[7]

And the last is RPOW Hal finney propose currency system based on a reusable proof of work (RPOW) in 2004[8].

Between 2008 and 2009, Bitcoin was made as to the first decentralized cryptocurrency by Satoshi Nakamoto. Nakamoto published the Bitcoin whitepaper 


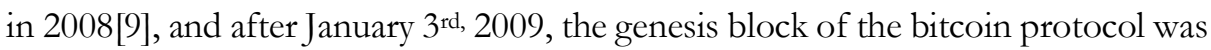
created. Nowadays it is most successful cryptocurrency in terms of market capitalization, beside above 700 altcoins that circulated in the world (eg. Litcoin, Etherum) based on Bitcoin have been proposed since the launch of Bitcoin.

\subsection{Cryptocurrency from Islamic Law Perspective}

The use of Bitcoin or other Cryptocurrency as a currency is also an interesting study from an Islamic economic perspective. The emergence of new phenomena in the economy must be followed by their compliance with Islamic law. The use of Bitcoin as a currency should be noted whether it is correct or not?

Different situation with Bitcoin or other common cryptocurrencies with the decentralization system and no regulation, in the Islamic rule, there is a need for monitoring organization of the cryptocurrency as stated by Abu-bakar [2]. Because when we talk about the Digital Money is still many issues about Dynamic Fluctuations, and influences by many uncertainties factors such as political, economic issue at impacted to local or global levels. Because of so many issues behind it, when Moslem countries trying to adopted or applied cryptocurrency as currency or investments it makes a problem that not in accordance with Islamic shariah-law.

Islam as a religion, which provides standard guidelines for Muslims in financial transactions, The rules and regulations regarding financial systems state that there should be no activities involving gambling (maysir), interest (riba) and uncertainty (gharar). Any activity related to one or more of the three aforementioned shariah injunctions is termed as forbidden [3]. termed as forbidden [3] The maysir, which is gambling is referred to as wagering of a valuable item such as money on an event with an indeterminate or uncertain outcome. The riba is factually seen as al-ziyadah, which is the addition of gain in a transaction without loss in the case of the failed business. Gharar means uncertainty due to deception and unclear risk involved in a transaction [2]. All these three concepts of the Islamic injunctions can be associated with the existing cryptocurrencies system since they are used as a store of value and medium of exchange.

The demand of the Islamic world cannot be ignored considering the immense growth of the global halal industry worldwide. Further, Islamic finance has reached up to one hundred and sixty-two trillion united states dollars (USD \$162 trillion) in the global commercial banking assets [10]

However, investment in the cryptocurrency market has been growing very slowly in the Islamic community. This is in connection with the unclear and 


p-ISSN: 2656-5935 http://journal-isi.org/index.php/isi e-ISSN: 2656-4882

dubious ruling of cryptocurrency, which is not within the sharia scope.

Consequently, many cryptocurrencies have been designed to address the limitation of the Bitcoin in the aspect of the sharia principles. The existing cryptocurrencies that claimed to be shariah-compliant includes OneGram, NoorCoin, Bayan Token, and Stellar cryptocurrency [11] . The one of hadits that that forms the basis of Cryptocurrency with shariah compliance, In the hadith of Ubadah bin Shamit radhiyallahu 'anhu. Rasulullah sallallaahu 'alaihi wa sallam said: "If gold is bartered with gold, silver is exchanged for silver, good wheat is exchanged for good wheat, coarse wheat is exchanged for coarse wheat, dates are exchanged for dates, salt is exchanged for salt, then the dose must be the same and cash. If the items being bartered are different then the measurements may be as you please as long as cash "(HR. Muslim 4147), they tried to use gold as a basis of value back their Digital Currency, which gold itself is famous for its exchange rate stability.

There is another problem as we mention above from abu bakar There is a need for monitoring organization of the cryptocurrency, to controlling from Riba (Interest), Maysir (gambling) and ghahar (Uncertainty). To solve this problem there is a need a tool that can predict with certainty based on valid historical data, to produce accurate prediction results and produce Economic value estimations that are close to Gold real value. With the results we can monitoring day by day, see next day value and continuously based on Cryptocurrency with value back by gold, and see what other impact influences the value by looking the factor negative or positive with sentiment analysis.

\subsection{Cryptocurrency Shariah}

In this chapter we will introduce, many kind of Cryptocurrency with based value back by gold that claimed to be shariah-compliant includes OneGram, NoorCoin, Hello Gold, Digix gold and Cyronium .

\subsubsection{Onegram}

OneGram is cryptocurrency that has been certified in compliance with called Shari'ah. It is inherently regulated by rules imposed on financial operations in the Islamic world.

OneGram Cryptocurrency is a digital currency supported by gold assets, respectively gram of gold represents one coin. The spot price of a digital currency depends on the time being gold market value. Thus, market use and demand adds a premium to value OneGram currency. Therefore, OneGram has a three assessment component to ensure the total market price. The first part is the Gold Value $(\mathrm{GV})$, which is determined by the spot price of gold. The second part is the 


p-ISSN: 2656-5935 http://journal-isi.org/index.php/isi e-ISSN: 2656-4882

current value of Transaction Costs (TF) which are reinvested to buy more gold, the value of which is determined by the level of utilization of OneGram coins. Then the third one the portion is the Economic Value (EV) determined by market demand. Therefore, the following formula in Eq. 1 is used to ensure market prices [12].

$$
\text { OneGram coin/token }(\mathrm{OGC}) \text { value }=\mathrm{GV}+\mathrm{EV}
$$

\subsubsection{NoorCoin}

The NoorCoin is designed based on Ziqilia blockchain leveraging smart contract technology. About Zilliqa is a high-throughput public blockchain platform designed to scale to thousands of transactions per second. The technology is a result of 3 years research at National University of Singapore, and NoorCoin w has been awarded a shariah certificate from an organization called world Sharia Advisory Committee.

Noorcoin will develop and implement the best transaction system that will be based on contracts that can be enforced without a third party between consumers and traders and a strong decentralized reputation management system. Transaction and fund transfer will be done in one step together with the applicable sales contract. Transaction costs will be simplified from many to simple transaction costs wherever consumers and traders are on the planet.

The Noor Coin transaction aspect imposes a transaction fee of $1.5 \%$ from investors, of which $0.5 \%$ of $1.5 \%$ is allocated to smart contract vouchers for investor token coins to use it in the ecosystem While $1 \%$ is assigned to the Noor Coin agency as income. This is according to sharia based on these conditions including; i) there is no usury (interest) in the issuance of Noor Coin ii) losses and profits are shared as part of the reward mechanism iii) there is lower speculation because cryptocurrency is supported by assets [13]. However, these assets are not mentioned in the white paper.

In fact, as mentioned earlier, cryptocurrency is not clearly supported by physical assets. So, the problem of uncertainty, speculation and volatility might be related to NoorCoin. Therefore, NoorCoin cannot be clearly considered a shariah compliant cryptocurrency in terms of uncertainty and volatility.

\subsubsection{Hellogold}

HGT is a token for supporting the objectives of the HelloGold Foundation. Supporters may be rewarded with any GOLDX distributed to HGT holders. It has no intrinsic value and is not backed by any real-world asset. A total one billion HGT has been minted. GOLDX is a token that is fully backed by $99.99 \%$ investment grade gold. 1 GOLDX is always tied to $1 \mathrm{~g}$ of gold, which is allocated and securely 


p-ISSN: 2656-5935 http://journal-isi.org/index.php/isi e-ISSN: 2656-4882

vaulted in Singapore. According to the company's website there will be three ways of receiving GOLDX in future: 1) provided as a reward for HGT holders by the HelloGold Foundation, 2) converting gold held as a customer of HelloGold into GOLDX [14].

\subsubsection{Digix gold}

DigixGlobal has created two types of Ethereum tokens - each with unique properties. DGX Digix Gold Token represents 1 gram of 99.99\% LMBA standard gold, secured in Safehouse vaults. Digix DAO Tokens (DGD) is used to claim quarterly rewards based on the total DGX collected through transaction fees. The DGD value fluctuates based on exchange rates and market forces. Digix tokenises gold on the Ethereum blockchain with our Proof of Provenance (PoP) protocol, which brings together verification by all third parties that we work with to ensure that the process is secure. DGX tokens are minted only upon the affixation of all signatures by our third party partners. The public blockchain further guarantees that records are unalterable and tamper-proof. [15]

\subsubsection{Cyronium}

Cyronium is a gold-backed crypto from Indonesia. Every 1 CYRO token has a guarantee of 1 Cyronium coin. 1 Cyronium coin consists of 20 grams of $99 \%$ gold LBMA (London Bullion Market Association). Where Cyronium differ from other offerings is they are offering the option of physical coins that will represent the CYRO token. If you elect to take the physical coin the CYRO token will be destroyed to avoid duplication. The coin will be shipped along with the certificate to the investor's address. Physical gold will be held in vaults in Singapore. [16]

\section{Overview of Economic value estimation Cryptocurrency in Computer Science Perspective}

There are many people doing research about value estimation using prediction of cryptocurrency. Greaves et al. [17] is propose technique using Logistic Regression and SVM and analyzed using Graph to estimated

The price of bitcoin. Huisu Jang et al. [18] they concern about study on modelling and prediction bitcoin with Bayesian Neural Network and giving some knowledge about bitcoin. Edwin sin et al. [19] provide topic Bitcoin price predictiom using Ensemble of Neural. Networks. Arief Radityo et al. [20] proposed a prediction of bitcoin using Artificial Neural Network Technique. They combine with market technical indicators but the results is worse of performance and training time. Sean et al [21] They propose method the price of Bitcoin using RNN and combine Using Reccurent Neural Network and Long Short Term Memory 


p-ISSN: 2656-5935 http://journal-isiorg/index.php/isi $\quad$ e-ISSN: 2656-4882

and Ruchi Mittal et al [22] is propose an Automated cryptocurrencies prices prediction using machine learning technique based on historical trend (daily trend).

Dibakar Raj Pant et al [23] They propose method prediction price of Bitcoin using RNN and combined using Sentiment analysis with good result and can see the impact of fluctuations value from negative or positive sentiments

The above researches proposed various method to prediction for bitcoin, in this paper, we analyze and study of knowledge about cryptcocurrency value back by gold, , technique and tools to predict Bitcoin or other cryptocurrency from many papers, internet and any other source and propose method to predict or estimate value for cryptocurrency value back based by gold.

\section{AN ANALYSIS OF CRYPTOCURRENCY VALUE ESTIMATION VALUE BACK BY GOLD}

\subsection{Cryptocurrency value estimation value back by gold.}

Technically Cryptcourrency value estimation value back by gold prediction it's the same with prediction technique on common fiat money or bitcoin but in the other way, when you try another technique and strategy like sentiment analysis maybe we can't get different results or the strategy it's not work. Because beside many factor that impact on common currency or stock exchange prediction, Bitcoin it's decentralized and not regulated by any party so it's different from common currency or common stock market.

We can use same algorithm to Bitcoin prediction using Machine Learning (eq. SVM, Naïve bayes, Regression) [17][18] or any other Advance Machine learning technique to improve the results like Deep Learning using Neural Network (eq. ANN and RNN)[20]

On Prediction we can predict Bitcoin using technique on specific subject that we wanted. Example, we want to predict only by the signal or the price, or we can predict just for current day or next day close value based on Long Short Term Memory (LSTM),[22] historical price and other technique like regime prediction to detect current day's trend on market, to help public people or company to investment using cryptocurrency or as currency exchange.

\subsection{Cryptocurrency value back by gold estimation Methods and Techniques}

To make more accurate and enrich the result we can combine the prediction algorithm with another method or technique on estimation cryptocurrencies In this section we describe some of technique that mention on previous research on some papers.

Market technical analysis is is a method that studies price movements by looking at historical price data that occur in the market through media charts. By 


p-ISSN: 2656-5935 http://journal-isi.org/index.php/isi e-ISSN: 2656-4882

studying this historical data a conclusion can be drawn for making investment decisions in the market.

There are several reasons why we should use TA, the first, not necessarily fundamental analysis can be applied in trading. I do not say Fundamental is not important, but here that needs to be underlined fundamental analysis will be far more important if our position as an Investor where the investment period can take more than one year.

Secondly, with the media chart it will be seen how the journey of a stock price where it is very helpful for traders to analyze in anticipation of future price changes and see patterns of patterns that occur in the price movements of a stock, so traders do not trade in ' darkness or without clear direction.[24]

Another technique to interpretation trend is Technical Indicator [25] is a series of data points obtained by applying the formula to securities price of data. The combinations of price data, such as close, low, high, low and open can be used as data point certain period of time. Some indicators can only be use on opening or closing prices, The price data is entered into the formula and a data point is produced. For Example, the average of 3 closing prices is one data point $((35200+25300+27550) / 3=29350)$.

A comparison can be made between present and past levels by creating a time series of data points. For analysis technical indicators are usually used to refine data points and displayed in a graphical form above or below by price, value and signal chart [9]. For maximize the dataset and predict some technical indicators such as simple moving average (SMA) is one of the simplest types of Moving Averages indicators for trading. Basically, the Simple Moving Average is calculated by summing the last few closing prices, here I call $\mathrm{X}$ period, and then dividing the amount by X. The result will be a moving average that changes over time, as long as prices are still raised by the market. Parabolic SAR (Stop and Reverse) method to find potential reversal in the mrket price prediction, Correlation (Corr) is a statistic that measures the degree to which two securities move in relation to each other, Average Directional Index (ADX) developed by J. Welles Wilder, is an indicator that can be used to determine the movement of when the market is trending, and how strong or weak the trend is and when the trend is likely to start or end [26], and Relative strength index (RSI) is an oscillator indicator with the lowest range (0) to the highest range (100). A range below 30 is called an oversold area and a range above 70 is called an overbought area.[27]. The advantages of using technical analysis it is used buy approximately $90 \%$ of the majir stock tradersm and it is also used to analyze the stock for shorter period.

Time series Data Analysis in the terms of future price predictions popular methods is using Autoregressive integrated moving average (ARIMA) models are a popular choice for forecasting over a short term conditions, it works when data 


p-ISSN: 2656-5935 http://journal-isi.org/index.php/isi e-ISSN: 2656-4882

exhibits consistent or stable pattern (constant) over time with least possible outliers. But this is not work always in the real time scenario, where data fluctuated drastically and it is highly volatile.[28]

Trading Strategy, this strategy is that we maintain position of +1 Bitcoin, 0 Bitcoin or -1 Bitcoin. At each time we predict the average price movement over the 10 seconds interval, say $\Delta \mathrm{p}$, using Bayesian regression (if $\Delta \mathrm{p}>\mathrm{t}$, a threshold, then we buy a bitcoin if current bitcoin position is $\leq 0$; if $\Delta \mathrm{p}<-\mathrm{t}$, then we sell a bitcoin if current position is $\geq 0$; else do nothing. The choice of time steps when make trading decisions as mentioned above are chosen carefully by looking at the recent trends. [29]

\subsection{Cryptocurrency value back by gold estimation tools}

Cryptocurrency estimation tools based on previous Research have increased from 2014 until 2019 many tools produces from authors that proposed using machine learning algorithm combine with some technique that we mention before. Their using many open source tools to produce the automated tools to bitcoin prediction, (Eq. Python, R, Weka, and Mathlab)

From Alex Greaves et al. 2015, their reseach propose prediction method using transaction graph to predict the price of bitcoin, their colled data from CS224 Website, and using feature Extraction and using SVM algorithm and Linear Regression to provide the results.

\begin{tabular}{lc}
\hline Regression Model & MSE \\
\hline Baseline & 2.02 \\
\hline Linear Regression & 1.94 \\
\hline \multicolumn{2}{c}{ Tabel 1 Regression results } \\
\hline \multicolumn{2}{c}{1.98} \\
\hline Classification Model & Accuracy \\
\hline Baseline & $53.4 \%$ \\
\hline Logistic Regression & $54.3 \%$ \\
\hline SVM & $53.7 \%$ \\
\hline Neural Network & $55.1 \%$ \\
\hline \multicolumn{2}{c}{ Table 2 Classification results }
\end{tabular}

Based on table 1 and 2 , we have different results provide from the author the first on regression model, the baseline have good result behind. Linear or SVM, and for classifcations the accuracy reach by Neural Network $55.1 \%$ by percentage. 
Hiusu jung et al. 2017, the authors provide empirical study and prediction of bitcoin using Bayesian neural network, and the result BNN give accurate prediction .

\begin{tabular}{llllll}
\hline \multicolumn{2}{l}{ Response Var. } & \multicolumn{2}{l}{ Log Price } & \multicolumn{2}{l}{ Log Volatility } \\
\hline \multicolumn{2}{l}{ Num . of Input var. } & 26 & 16 & 25 & 16 \\
\hline Linear & RMSE & - & 0.0913 & - & 0.4595 \\
\cline { 2 - 6 } Regression & MAPE & - & 0.0681 & - & 0.5905 \\
\hline Bayesain & RMSE & 0.0031 & 0.0047 & 0.1612 & 0.1717 \\
\cline { 2 - 6 } NN & MAPE & 0.0119 & 0.0148 & 0.33 .14 & 0.3512 \\
\hline Support & RMSE & 0.1453 & 0.1434 & 0.3810 & 0.3939 \\
\cline { 2 - 6 } $\begin{array}{l}\text { Vec. } \\
\text { Regression }\end{array}$ & MAPE & 0.0325 & 0.0322 & 0.5411 & 0.6293 \\
\hline
\end{tabular}

Tabel 3 Trainning error for the bitcoin price information

\begin{tabular}{llllll}
\hline \multicolumn{2}{l}{ Response Var. } & \multicolumn{2}{l}{ Log Price } & \multicolumn{2}{l}{ Log Volatility } \\
\hline \multicolumn{2}{l}{ Num . of Input var. } & 26 & 16 & 25 & 16 \\
\hline Linear & RMSE & - & 0.0935 & - & 0.4823 \\
\cline { 2 - 6 } Regression & MAPE & - & 0.0712 & - & 0.6263 \\
\hline Bayesain & RMSE & 0.0039 & 0.0069 & 0.2546 & 0.2325 \\
\cline { 2 - 6 } NN & MAPE & 0.0138 & 0.0180 & 05090 & 0.5222 \\
\hline $\begin{array}{l}\text { Support } \\
\text { Vec. }\end{array}$ & RMSE & 0.3201 & 0.2742 & 0.5487 & 0.5297 \\
\cline { 2 - 6 } Regression & MAPE & 0.0428 & 0.0404 & 0.7232 & 0.8629 \\
\hline
\end{tabular}

Table 4 Test error for the bitcoin price information

Based on table 3 From this results, we can confirm that Bayesian NN is show good result and better apply for the Bitcoin prediction. time series analysis and Support vector regression shows not good performances in both training and test phase.

Arief Radityo et al. 2017, the authors provide prediction of bitcoin exchange rate using Artifical neural network combine with technical analysis and this is the results.

\begin{tabular}{ccc}
\hline Methods & MAPE $\%$ & $\begin{array}{c}\text { Training Time } \\
\text { (seconds) }\end{array}$ \\
\hline BPNN & $1.998 \pm 0.038$ & $347 \pm 63$ \\
\hline GANN & $4.461 \pm 0.49$ & $467 \mathrm{v} 345$ \\
\hline GABPNN & $1.883 \pm 0.066$ & $1539 \pm 558$ \\
\hline NEAT & $2.175 \pm 0.096$ & $470 \pm 363$ \\
\hline
\end{tabular}

Tabel 5 Experiment result Arief radityo 


P-ISSN: 2656-5935 http://journal-isi.org/index.php/isi e-ISSN: 2656-4882

From Table I, the result shown that GABPNN has the best accuracy with average of MAPE $1.883 \%$. The worst accuracy is GANN with average MAPE $4.461 \%$ and GABPNN has the longest training time.

Sean Mcnally et al. 2018 provide method prediction bitcoin using Bayesian optimised recurrent neural network (RNN) and a Long short term memory (LSTM).the LSTM achives the highest classification accuracy above 52\% and a RSME of $8 \%$

\begin{tabular}{ccccc}
\hline Model & Length & Precision & Accuracy & RMSE \\
\hline LSTM & 100 & $35.50 \%$ & $52.78 \%$ & $6.78 \%$ \\
\hline RNN & 20 & $39.08 \%$ & $50.25 \%$ & $5.45 \%$ \\
\hline ARIMA & 170 & $100 \%$ & $50.05 \%$ & $53.74 \%$ \\
\hline
\end{tabular}

Table 6. Model Results

Dibakar Raj Pant et al. 2018 Provide method to estimation bitcoin price using Reccurent Neural Network and Sentiment analysis. Propose method that they provide is unique, The major contribution of this work is a sentiment analyserwhich can distinguish between the positive and negative tweets of Bitcoin over the Twitter with the accuracy of $81.39 \%$ shown on table 7. Further the RNN model which can predict the price of Bitcoin the following day taking consideration of historical price and the positive and negative sentiment scores with the accuracy of $77.62 \%$ is another useful work. It discusses the different methods of feature extraction and provides a comparison between them. It also shows a moderate correlation of 0.41 between rise of negative opinions in the Twitter related toBitcoin and its consequent fall in price.

\begin{tabular}{cccc}
\hline Accuracy & Precision & Recall & F-Measure \\
\hline $81.3 \%$ & $82.90 \%$ & $84.86 \%$ & $83.86 \%$ \\
\hline \multicolumn{4}{c}{ Table 7. Model Results } \\
\hline \multicolumn{4}{c}{}
\end{tabular}

\begin{tabular}{ll}
\hline Price Fluctuations & Person Correlation Coefficient \\
\hline More than $2 \%$ & Negative Sentiment 0,34 \\
\cline { 2 - 2 } & Positive Sentiment 0.21 \\
\hline More than $4 \%$ & Negative Sentiment 0,41 \\
\cline { 2 - 2 } & Positive Sentiment 0.26 \\
\hline
\end{tabular}

Table 8. Sentiment and Price Corellation Results

\section{FUTURE DIRECTIONS OF CRYPTOCURRENCY VALUE ESTIMATION VALUE BACK BY GOLD}

188 | A Study of Economic V alue Estimation on Cryptocurrency .... 


p-ISSN: 2656-5935 http://journal-isi.org/index.php/isi e-ISSN: 2656-4882

From some previous research paper their produce good results and good techniques to provide Cryptocurrency estimation to help the investor to make decision of investment in cryptocurrency

With various algorithm and various results, in the Arief radityo research they tried to combine Advance technique using deep learning and combine with market technical analysis, the result is more rich than other, but disadvantage their not mention the result is good or not to help investor to make decision, maybe because their using MAPE to evaluate the results, Dibakar Raj Pant et al. 2018 Provide method to estimation bitcoin price using Recurrent Neural Network and Sentiment analysis It also shows a moderate correlation of 0.41 between rise of negative opinions in the Twitter-related to bitcoin and its consequent fall in price. The proposed method that they provided is unique

Future works will be, the direction will optimize the dataset shrouded the noise, use some feature, and make shorter training time, combine with some technique and algorithm (Hybrid methods) we can select from the previous research that provide good results, Eq. BNN with Market Technical Analysis and LSTM using Historical price data and combine with sentiment analysis to provide new best results and fix the ghahar (Uncertainty) in Islamic Law.

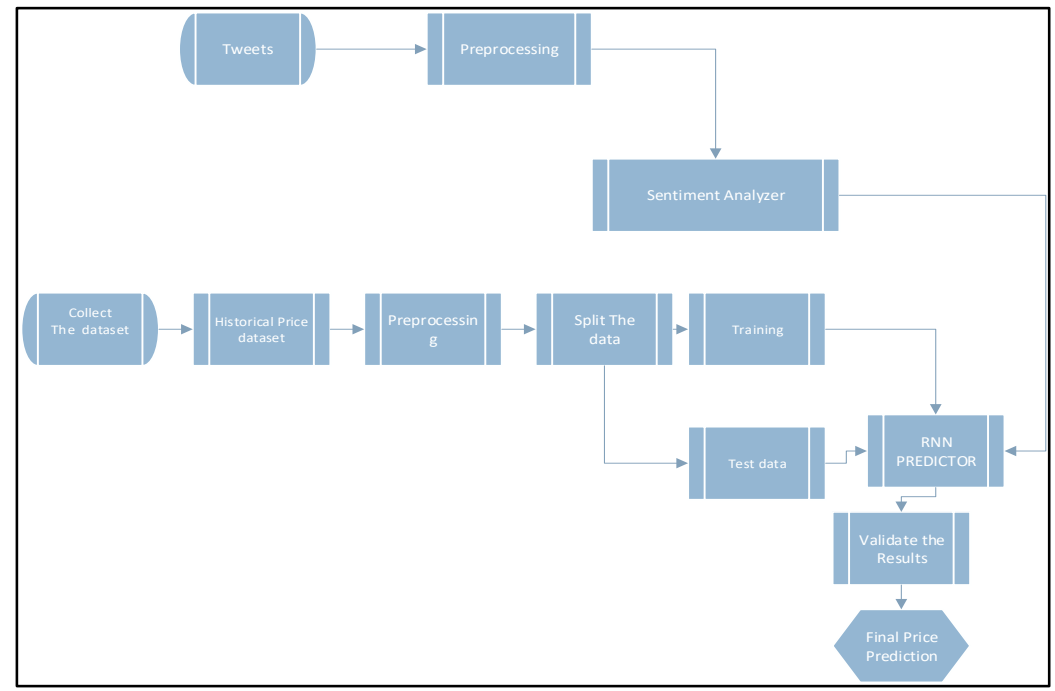

Figure 1. Estimation Method to predict the Cryptocurrency Value

Based on Figure 1, we provide method to predict cryptocurrency value enhance from previous research using historical price data combine with sentiment analysis to see impact value and validate the results to find best results before finalize the price prediction. 


p-ISSN: 2656-5935 http://journal-isi.org/index.php/isi e-ISSN: 2656-4882

\section{CONCLUSION AND FUTURE WORKS}

Afterward, as we mentioned before in the article, the price of cryptocurrency is influenced by many uncertainty factors. The prices are influenced by many uncertainties factor such as political issue, the economic issue at impacted to local or global levels. So prediction price bitcoin using LSTM can't good enough to make the decision to invest in cryptocurrency value back by gold, it is another side for taking the decisions.

But in some way it can fix some problem in Islamic Perspective for Muslims Country and follow the rules, The problem as we mention above from abu bakar There is a need for monitoring organization of the cryptocurrency, to controlling from Riba (Interest), Maysir (gambling) and ghahar (Uncertainty). To solve this problem there is a need a tool that can predict with certainty based on valid historical data, to produce accurate prediction results and produce Economic value estimations that are close to Gold real value. With the results we can monitoring day by day, see next day value and continuously based on Cryptocurrency with value back by gold, and see what other impact influences the value by looking the factor negative or positive with sentiment analysis.

Future research will focus on, modified LSTM layers, adding dropout and modified number of epochs, and using different instability dataset to test how good the prediction results or try to use sentiment analysis combined with LSTM method to see the impact of the uncertainty in value bitcoin.

\section{REFERENCES}

[1] G. Hileman, "State of blockchain q1 2016: Blockchain funding overtakes bitcoin," CoinDesk, New York, NY, May, vol. 11, 2016.

[2] Y. S. Abubakar, A. F. Ogunbado, and M. A. Saidi, "Bitcoin and its Legality from Shariah Point of View," SEISENSE J. Manag., vol. 1, no. 4, pp. 1321, 2018.

[3] A. K. M. Meera and others, "Cryptocurrencies From Islamic Perspectives: The Case Of Bitcoin,” Bull. Monet. Econ. Bank., vol. 20, no. 4, pp. 1-18, 2018.

[4] A. Judmayer, N. Stifter, K. Krombholz, and E. Weippl, "Blocks and Chains: Introduction to Bitcoin, Cryptocurrencies, and Their Consensus Mechanisms," Synth. Lect. Inf. Secur. Privacy, Trust, 2017.

[5] W. Dai, "B-money proposal," White Pap., 1998.

[6] N. Szabo, "Bit gold," Website/Blog, 2008.

[7] A. Back, "Hashcash." 1997.

[8] Hal Finney, "RPOW - Reusable Proofs of Work," Agosto 15, 2004. 2004.

[9] S. Nakamoto and others, "Bitcoin: A peer-to-peer electronic cash system," 2008.

[10] Bayan token, “A Shariah-Compliant Utility Crypto Token,” 2018. [Online].

190 | A Study of Economic V alue Estimation on Cryptocurrency .... 


p-ISSN: 2656-5935 http://journal-isi.org/index.php/isi e-ISSN: 2656-4882

Available:

https://www.bayantoken.com/files/frontend/BayanatTokenWhitepaper -v3.10.pdf. [Accessed: 29-Aug-2019].

[11] A. Aliyu et al., "Review of Existing Shariah-Compliant Cryptocurrency," in INHAC 2019, 2019.

[12] Onegram, "OneGram Whitepaper," 2017. [Online]. Available: https://onegram.org/whitepaper. [Accessed: 20-Aug-2019].

[13] NoorCoin, "Noorcoin white paper," 2018. [Online]. Available: https://www.noorcoin.io/NoorcoinWhitepaper.pdf.\%0A. [Accessed: 20Aug-2019].

[14] HelloGold, "HelloGold," 2017. [Online]. Available: https://www.allcryptowhitepapers.com/wpcontent/uploads/2018/05/HelloGold-Whitepaper.pdf. [Accessed: 20Aug-2019].

[15] Digix Global, “No Title," 2016. [Online]. Available: https://digix.global/whitepaper.pdf. [Accessed: 20-Aug-2019].

[16] Cyronium, "Cyronium Whitepaper." [Online]. Available: https:// docplayer.info/80889033-Cyronium-whitepaper-cyronium-thefirst-cryptocurrency-with-precious-metal-underlying.html. [Accessed: 20Aug-2019].

[17] A. Greaves and B. Au, "Using the bitcoin transaction graph to predict the price of bitcoin," No Data, 2015.

[18] H. Jang and J. Lee, "An Empirical Study on Modeling and Prediction of Bitcoin Prices With Bayesian Neural Networks Based on Blockchain Information," IEEE ACCESS, vol. 6, pp. 5427-5437, 2018.

[19] E. Sin and L. Wang, "Bitcoin Price Prediction Using Ensembles of Neural Networks," in 2017 13TH INTERNATIONAL CONFERENCE ON NATURAL COMPUTATION, FUZZY SYSTEMS AND KNOWLEDGE DISCOVERY (ICNC-FSKD), 2017, pp. 666-671.

[20] A. Radityo, Q. Munajat, and I. Budi, "Prediction of Bitcoin exchange rate to American dollar using artificial neural network methods," in Advanced Computer Science and Information Systems (ICACSIS), 2017 International Conference on, 2017, pp. 433-438.

[21] S. McNally, J. Roche, and S. Caton, "Predicting the price of Bitcoin using Machine Learning," in Parallel, Distributed and Network-based Processing (PDP), 2018 26th Euromicro International Conference on, 2018, pp. 339-343.

[22] R. Mittal, S. Arora, and M. P. S. Bhatia, "AUTOMATED CRYPTOCURRENCIES PRICES PREDICTION USING MACHINE LEARNING," 2018.

[23] D. R. Pant, P. Neupane, A. Poudel, A. K. Pokhrel, and B. K. Lama, "Recurrent Neural Network Based Bitcoin Price Prediction by Twitter Sentiment Analysis," in 2018 IEEE 3rd International Conference on Computing, Communication and Security (ICCCS), 2018, pp. 128-132. 
[24] J. J. Murphy, "Technical Analysis Of The Financial Markets," Pennsylvania Dental Journal. 1999.

[25] E. Kristensen, S. Østergaard, M. A. Krogh, and C. Enevoldsen, "Technical Indicators of Financial Performance in the Dairy Herd," J. Dairy Sci., 2008.

[26] J. W. Wilder, New concepts in technical trading systems. Trend Research, 1978.

[27] Investopedia, “Trading Strategy," 2018. [Online]. Available: https://www.investopedia.com/. [Accessed: 14-Mar-2019].

[28] C. Scheier and W. Tschacher, "Appropriate algorithms for nonlinear time series analysis in psychology," in Nonlinear dynamics in buman behavior, World Scientific, 1996, pp. 27-43.

[29] D. Shah and K. Zhang, "Bayesian regression and Bitcoin," in 2014 52nd Annual Allerton Conference on Communication, Control, and Computing (Allerton), 2014, pp. 409-414. 